
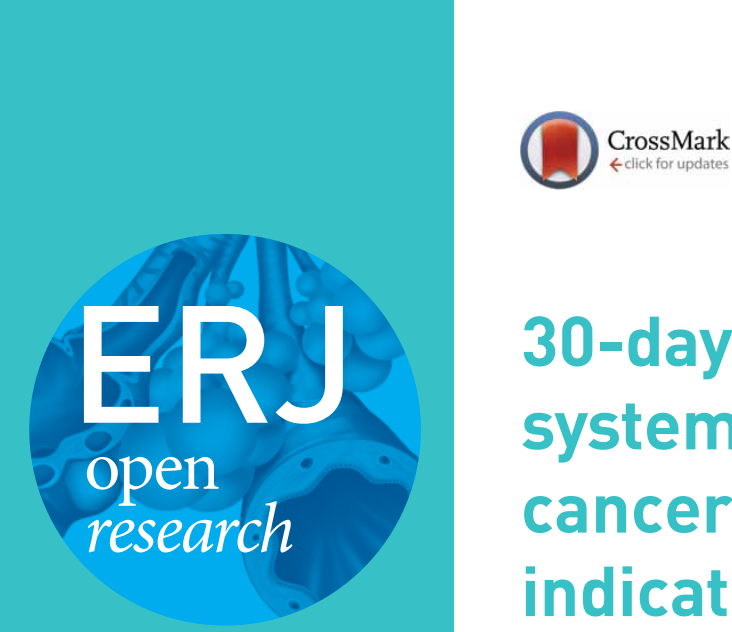

\title{
30-day mortality after the start of systemic anticancer therapy for lung cancer: is it really a useful performance indicator?
}

\author{
Jacobus Adrianus Burgers ${ }^{1}$ and Ronald Alphons Damhuis ${ }^{2}$
}

Affiliations: ${ }^{1}$ Dept of Thoracic Oncology, Netherlands Cancer Institute, Amsterdam, The Netherlands. ${ }^{2}$ Dept of Research, Netherlands Comprehensive Cancer Organisation, Utrecht, The Netherlands.

Correspondence: J.A. Burgers, Dept of Thoracic Oncology, Netherlands Cancer Institute, Plesmanlaan 121, 1066 CX Amsterdam, The Netherlands. E-mail: s.burgersanki.nl

ABSTRACT Systemic treatment is the standard treatment for unresectable stage III and IV lung cancer. Nevertheless, a $5-10 \%$ death rate has been described within 30 days after the last systemic treatment, suggesting that these patient did not benefit. We analysed the 30-day mortality after start of systemic therapy.

Data were retrieved from the Netherlands National Cancer Registry. From 2010 to 2015, 26277 patients were included. $56 \%$ were men. The median age was 65 years and $31 \%$ of patients were aged $\geqslant 70$ years. $27 \%$ involved small cell lung cancer and $73 \%$ nonsmall cell lung cancer. Overall mortality within 30 days after the start of systemic treatment was $6.2 \%$. Multivariable analysis established the prognostic influence of age, histology, number of metastatic sites and type of systemic treatment. Chemotherapy was administered in 77 hospitals, treating each 15-161 lung cancer patients with systemic therapy annually. None of the hospitals had a significantly higher 30-day mortality according to hierarchical multivariable analysis, controlling for case-mix.

In the Netherlands, the 30-day mortality rate after start of systemic therapy for lung cancer patients was comparable with earlier reports. Hospital volume did not influence the 30-day mortality rate. 30-day mortality rate is not a meaningful indicator to monitor quality of care.

@ERSpublications

30-day mortality rate after first-line systemic therapy for lung cancer does not seem to be a meaningful indicator to monitor quality of care http://ow.ly/2cWn30lDlpW

Cite this article as: Burgers JA, Damhuis RA. 30-day mortality after the start of systemic anticancer therapy for lung cancer: is it really a useful performance indicator? ERJ Open Res 2018; 4: 000302018 [https://doi.org/10.1183/23120541.00030-2018].

Received: Feb 142018 | Accepted after revision: Aug 242018

Copyright $\odot$ ERS 2018. This article is open access and distributed under the terms of the Creative Commons Attribution Non-Commercial Licence 4.0. 


\section{Introduction}

Lung cancer has a dismal prognosis, notwithstanding the exciting progress that has been made in the fields of stereotactic irradiation, personalised medicine and immunotherapy. In advanced-stage lung cancer, treatment focuses on prolonging the remaining time of life, while preserving an as well as possible quality of life. The balance in doing right or wrong remains delicate, especially when chemotherapy is prescribed.

The importance of this balance is underlined by the limited survival of stage IV lung cancer patients. In 2004, the Big Lung Trial settled the question of whether chemotherapy improved survival in nonsmall cell lung cancer (NSCLC) patients [1]. Median survival improved from 5.7 to 8.0 months in the best supportive care and chemotherapy groups, respectively. Patients with an activating epidermal growth factor receptor (EGFR) mutation have a more favourable median survival of 18.6 months, when treated with EGFR-tyrosine kinase inhibitors [2]. Second-line treatment with docetaxel improved the median survival from 4.6 months for patients receiving best supportive care to 7.0 months for those receiving docetaxel [3] In general, chemotherapy prolongs overall survival and symptom-free survival, it palliates tumour-related symptoms and improves quality of life [4]. Unfortunately, not every patient will benefit.

It is up to the physician to estimate whether a patient is fit for the treatment and likely to benefit from systemic therapy. Guidelines list good performance status and sufficient organ function as predictors for uneventful chemotherapy [5,6]. Advanced age is not regarded as a discriminating factor.

Apart from survival time, patient-reported outcome measures are becoming increasingly important in the assessment of treatments and clinics [7] The International Consortium for Health Outcomes Measurement advocates the use of quality-of-life and quality-of-death measures. The latter focus on the dismal sides of treatment. Parameters like "place of death" and "duration of time spent in hospital at the end of life" make quality of death a measurable parameter. This stresses the need for proper selection of patients for chemotherapeutic treatment, in order to minimise severe toxicity, leading to hospitalisation and early death.

In 2016, 30-day mortality after systemic anticancer treatment was proposed as a novel indicator to monitor the quality of care [8]. The 30-day cut-off seems appropriate, as patients who succumb within this short period of time are not likely to have benefited from any systemic therapy.

In England, National Health Service hospital trusts routinely report key treatment details to Public Health England, including the treatment regimen, start date and cycle number. For a cohort of 15045 lung cancer patients who received systemic therapy in 2014, 30-day mortality was calculated from the start of the most recently reported treatment cycle. Mortality was $8 \%$ overall, ranging from 3\% in patients treated with curative intent to $10 \%$ in patients treated with palliative intent. Recently, 30-day mortality in a Canadian Cancer Centre was reported for NSCLC patients treated from 2010 to 2014 [9]. The 30-day mortality for cytotoxic chemotherapy was $10.6 \%$, for anaplastic lymphoma kinase/tyrosine kinase inhibitor therapy $10.3 \%$

Considering that this $10 \%$ figure is deemed unexpectedly high, we decided to repeat the study in patients with advanced-stage lung cancer in the Netherlands. Potential prognostic factors were analysed to identify subgroups at higher risk of early death and to assess variation between individual hospitals and hospital characteristics.

\section{Methods}

Source

Information regarding lung cancer patients who underwent systemic treatment for TNM (tumour, node, metastasis) stage III/IV from 2010 to 2015 was retrieved from the Netherlands National Cancer Registry (NNCR), after approval by the privacy review board. In accordance with the regulations of the Central Committee on Research Involving Human Subjects, this type of study does not require approval from an ethics committee in the Netherlands. The NNCR collects data on all cancer patients diagnosed in the Netherlands, based on notification of newly diagnosed malignancies by the national automated pathological archive and of hospital discharge diagnoses. Information on demographics, diagnosis, staging and treatment is extracted routinely from the medical records by specially trained NNCR personnel. Stage information was recorded according to the 7th edition of the TNM Classification of Malignant Tumors from the International Union Against Cancer. Information on survival status and date of death is updated annually using a computerised link with the national civil registry. The starting date of systemic treatment is manually recorded but information on consecutive cycles, and maintenance or second-line treatment is lacking. Information on chemotherapy regimen, performance status and/or comorbidity was not available. 


\section{Patient selection}

This study incorporated adult patients (aged $>17$ years) with stage III or IV lung cancer, irrespective of the histological subtype, and primarily treated with systemic therapy (excluding hormonal treatment and immunotherapy). The selection process included patients who later received additional treatment such as radiotherapy or surgery. Records were excluded if the date of systemic treatment was missing $(\mathrm{n}=748)$ or when systemic treatment was not the first therapy $(n=2315)$.

\section{Study variables}

Age was categorised in four subgroups. Histology is coded according to the third edition of the International Classification of Disease for Oncology (ICD-O-3) and was categorised in four subgroups: small cell lung cancer (SCLC), squamous cancer, adenocarcinoma and a remaining group comprising large cell carcinoma, not otherwise specified and tumours such as carcinoids and sarcomatoid carcinomas. Stage was stratified as IIIA, IIIB and IV. For patients with stage IV disease, the number of metastatic sites involved was designated as one, two or three or more. Type of treatment was categorised as chemotherapy or targeted treatment.

\section{Statistical analysis}

Statistical analyses were performed using Stata 14 (StataCorp, College Station, TX, USA). The proportion of patients with 30-day mortality after start of systemic treatment was tabulated and differences between subgroups were tested for significance using Chi-squared statistics. Multivariable analysis was performed using logistic regression. The significance of predictive factors was assessed in backward analysis with the log-likelihood test and significant $(\mathrm{p}<0.05)$ parameters are represented by OR $(95 \% \mathrm{CI})$. Variation between hospitals was assessed using hierarchical logistic regression, including hospital of chemotherapy as a first-level (random effect) variable. Patients were excluded if the hospital of chemotherapy was unknown $(n=19)$ or when the patient was treated abroad $(n=31)$. Odds ratios for the involved hospitals were derived by post-estimation commands and are plotted against volume. Significant aberrations $(\mathrm{p}<0.05)$ are displayed by a marker with a different type and colour. The hospital with the largest treatment volume is not included in the graph to preserve anonymity.

\section{Results}

The study series comprised 26277 patients, with more males (56\%) than females (44\%) (table 1). The median age was 65 years and $31 \%$ of patients were aged $\geqslant 70$ years. $27 \%$ were diagnosed with SCLC and $73 \%$ with NSCLC. Adenocarcinoma was more common than squamous carcinoma (41\% versus $17 \%$, respectively). TNM stage IV comprised two-thirds of the study population and $13 \%$ of cases had distant metastases in three or more organ systems. The proportion of patients treated with targeted treatment increased with time from $4 \%$ in 2010 to $7 \%$ in 2015 (data not shown).

Overall mortality within 30 days after the start of systemic treatment was $6.2 \%$. Only $11(0.04 \%)$ patients died on the first day; 313 (1.2\%) during the first week. Age, sex, stage, histology, the number of involved metastatic organs and type of systemic treatment were significantly correlated with 30 -day mortality rates in bivariate analysis. The 30-day mortality rose with increasing age from $4.6 \%$ in patients aged $18-59$ years to $9.8 \%$ in patients aged $\geqslant 80$ years. 30 -day mortality was particularly high for stage IV (7.8\%), SCLC $(8.5 \%)$, targeted treatment $(8.6 \%)$ and with distant metastases in three or more organ systems $(11.5 \%)$. Year of diagnosis had no influence on the 30-day mortality rate. Multivariable analysis confirmed the prognostic influence of age, histology, number of metastatic sites involved and type of systemic treatment. The risks were highest for patients aged $\geqslant 80$ years (OR 2.3, 95\% CI 1.8-2.9), SCLC (OR 1.7, 95\% CI 1.5-1.9), three or more metastatic sites involved (OR 4.3, 95\% CI 3.7-5.1) and targeted treatment (OR 1.3, 95\% CI 1.1-1.6). The risk in octogenarians with SCLC and three or more metastatic sites involved was $28 \%$.

Chemotherapy was administered in 77 hospitals, including one oncological centre, eight university hospitals and 68 general hospitals. The annual number of patients treated by each hospital ranged from 15 to 161. None of the hospitals had significantly higher 30-day mortality according to hierarchical multivariable analysis, controlling for case-mix (figure 1). In two hospitals, results were significantly better than the national average.

\section{Discussion}

Our study confirms a substantial risk of early death after systemic treatment for advanced lung cancer. Calculated from the start of the first cycle, the overall 30-day mortality was $6.2 \%$. This figure may seem low compared to other published data, but direct comparisons are problematic due to differences in study population and methodology. For example, the $10 \%$ rate reported for systemic treatment with palliative intent in England [8] not only involved first-line treatment, but included maintenance and second-line 


\begin{tabular}{|c|c|c|c|c|}
\hline & Subjects & 30-day mortality ${ }^{\#}$ & $p$-value & OR $(95 \% \mathrm{CI})$ \\
\hline \multicolumn{5}{|l|}{ Age years } \\
\hline $18-59$ & $7420(28)$ & 4.6 & \multirow[t]{4}{*}{$<0.001$} & 1 \\
\hline $60-69$ & $10555(40)$ & 6.0 & & $1.3(1.2-1.5)$ \\
\hline $70-79$ & $7163(27)$ & 7.5 & & $1.7(1.5-2.0)$ \\
\hline$\geqslant 80$ & $1139(4)$ & 9.8 & & $2.3(1.8-2.9)$ \\
\hline \multicolumn{5}{|l|}{ Year of diagnosis } \\
\hline 2010 & $4253(16)$ & 6.4 & \multirow[t]{6}{*}{0.15} & \\
\hline 2011 & $4254(16)$ & 7.0 & & \\
\hline 2012 & 4389 (17) & 6.2 & & \\
\hline 2013 & 4364 (17) & 6.1 & & \\
\hline 2014 & 4475 (17) & 5.6 & & \\
\hline 2015 & 4542 (17) & 6.0 & & \\
\hline \multicolumn{5}{|l|}{ Sex } \\
\hline Male & $14719(56)$ & 6.5 & \multirow[t]{2}{*}{0.03} & \\
\hline Female & $11558(44)$ & 5.8 & & \\
\hline \multicolumn{5}{|l|}{ Stage } \\
\hline IIIA & 4515 (17) & 2.5 & \multirow[t]{3}{*}{$<0.001$} & ๆ \\
\hline IIIB & $4330(16)$ & 3.6 & & \\
\hline IV & $17432(66)$ & 7.8 & & \\
\hline \multicolumn{5}{|l|}{ Histology } \\
\hline Adenocarcinoma & $10883(41)$ & 5.3 & \multirow[t]{4}{*}{$<0.001$} & 1 \\
\hline Squamous & 4414 (17) & 4.4 & & $1.1(0.9-1.3)$ \\
\hline Other NSCLC & $3770(14)$ & 6.5 & & $1.4(1.2-1.6)$ \\
\hline SCLC & 7210 (27) & 8.5 & & $1.7(1.5-1.9)$ \\
\hline \multicolumn{5}{|c|}{ Metastatic sites involved $\mathbf{n}$} \\
\hline 0 & 8841 (34) & 3.0 & \multirow[t]{4}{*}{$<0.001$} & 1 \\
\hline 1 & 9182 (35) & 6.0 & & $2.1(1.8-2.4)$ \\
\hline 2 & 4865 (19) & 8.6 & & $3.0(2.6-3.5)$ \\
\hline$\geqslant 3$ & 3389 (13) & 11.5 & & $4.3(3.7-5.1)$ \\
\hline \multicolumn{5}{|l|}{ Type of treatment } \\
\hline Chemotherapy & 25010 (95) & 6.1 & \multirow[t]{2}{*}{$<0.001$} & 1 \\
\hline Targeted & $1267(5)$ & 8.6 & & $1.3(1.1-1.6)$ \\
\hline
\end{tabular}

Data are presented as $\mathrm{n}(\%)$ or $\%$, unless otherwise stated. NSCLC: non-small cell lung cancer; SCLC: small cell lung cancer. " : as percentage of the population within this group; ": not included in multivariable analysis to avoid collinearity.

treatments, and it was measured from the last cycle of chemotherapy. The English study mentioned that the 30-day mortality was 2.5 times higher in treatment-naive patients, a population that more closely resembles the population in our analysis. In addition, direct comparison with end-of-life studies, which trace back treatment data for patients after death, is questionable. A recent French study evaluated $>270000$ hospitalised patients who died from metastatic cancer and reported that $20 \%$ received chemotherapy during the last month before death [10]. Again, this involves early death after any cycle, whereas our series only evaluated the first cycle. Similar disparities may occur by comparing data from population-based series with those from expert centres or trial populations. However, a combined analysis of clinical trials by the Medical Research Council Lung Cancer Working Party on small-cell lung cancer revealed that $10 \%$ of patients died within 3 weeks of the start of chemotherapy [11].

Irrespective of the definitions used, the general impression emerges that it will remain a challenge to determine which patients may benefit from chemotherapy, and for which patients treatment will merely be a burden during the last weeks of their life. This question not only relates to the start of chemotherapy, but also pertains to continuation of treatment for patients who experience toxicity, or to the decision to start with second-line chemotherapy or experimental treatment. Early death after systemic therapy, focussing on longer-term goals, implies that the victims may not have been prepared for their upcoming decease, and that they may not have had the time and opportunity for a proper farewell of their loved ones. Also, they may not have died in their preferred place of death. $>50 \%$ of patients, but also of relatives and friends, preferred home care in advanced illness, with inpatient hospice as a second choice [12]. This was confirmed in a prospective telephone survey of $>9500$ people in seven Western European 
FIGURE 1 Residual between-hospital variation in 30-day mortality after the start of systemic treatment in patients with stage III-IV nonsmall cell lung cancer.

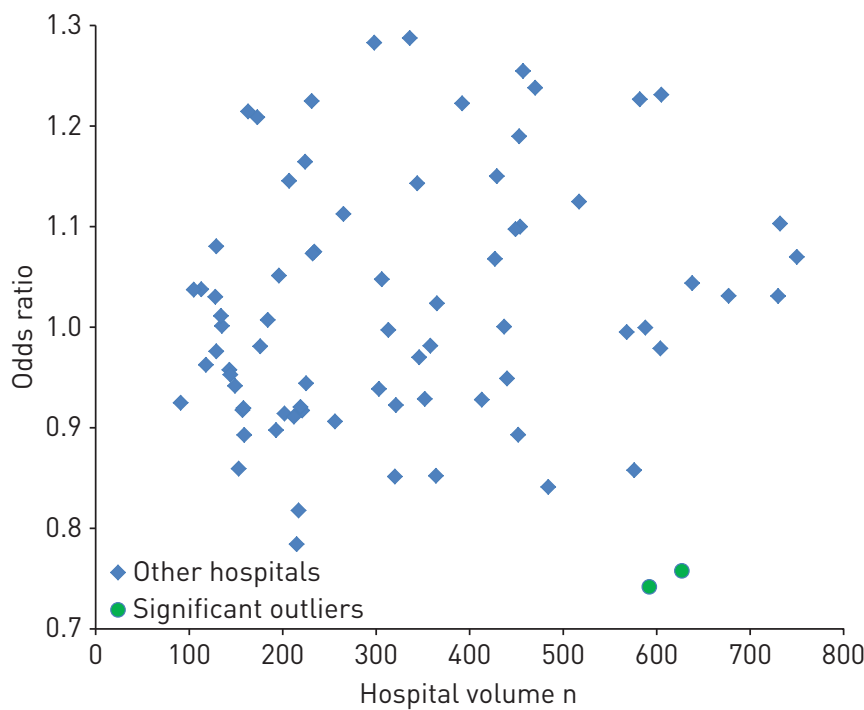

countries [13], in which $51-84 \%$ of the participants mentioned that they would prefer to die at home. This contrasts with the observation that $22-52 \%$ of the cancer patients aged $>65$ years, included in a retrospective cohort study using administrative and registry data from 2010 from seven countries, died in an acute-care hospital [14].

Our analysis identified several factors predicting a higher 30-day mortality, such as high age, high tumour stage and small cell lung cancer histology (versus NSCLC). These factors are well-known prognostic factors too. Our study design does not give us the opportunity to discriminate between factors associated with higher chemotherapy-induced mortality and prognostic factors identifying patients with a poor prognosis, irrespective of the treatment. The 30-day mortality might be unexpectedly high due to our inability to properly identify patients with a poor short-term prognosis. For individual decision making, better prognostic models are needed, including parameters on comorbidity and performance score [15].

Although systemic therapy is the standard treatment for advanced stage lung cancer, thus far early death has received little attention. Early death can be related to treatment-related toxicity or to progressive disease. In our study, the cause and place of death might have shed more light on this subject, but these data were not available due to privacy regulations. In general, neutropenic sepsis generally occurring at day 10-15 may be responsible for death within 30 days. A study from the Royal Marsden Hospital (London, UK) evaluated all patients who received chemotherapy and assessed the cause of death for patients who died within 30 days [16]. They reported an $8 \%$ mortality and concluded that $76 \%$ of deaths were related to progressive disease. This supports the above-mentioned statement that the 30 -day mortality is probably mainly influenced by the natural course of lung cancer.

The high 30-day mortality rate in patients receiving targeted therapy is hard to explain, as this treatment tends to have a higher radiological and clinical response rate than chemotherapy for the selected patient population [2]. Whether this can be explained by patient mix, allowing patients with a worse performance to start this type of treatment, remains to be established. A fact is that, even within selected EGFR-mutated study populations, lung cancer patients do die within 30 days after start of therapy.

Currently, immunotherapy has been registered as first-line systemic treatment [17]. Immunotherapy combinations [18] and combinations of immunotherapy with chemotherapy have shown progression-free survival and/or survival benefits $[19,20]$. A closer look to the survival curves of the phase III studies revealed a 30-day mortality estimate of 3-8\%. Early death after the start of therapy will remain a challenge for the near future, and when immunotherapy combinations become standard of care.

Our analyses did not show major institutional variation, suggesting that early death after systemic therapy is a universal phenomenon affecting every hospital. Results were superior in three out of 77 hospitals, perhaps related to clinical care or better/more stringent selection of fit patients. Given the absence of information on performance status or comorbidity, residual confounding may still be present and 30-day mortality might be lower in hospitals where doctors are more reluctant to prescribe systemic treatment. In our series, 6 years of data were needed to detect three outliers and, hence, 30-day mortality does not appear to be a promising quality parameter at hospital level. It does not fulfil the criteria for a worthwhile parameter, able to discriminate good from poor quality of care [15]. Such a parameter should be sensitive, 
needs to be robust, accurate, easily reproducible and interpretable, and validated in the relevant setting. In England, the figures from individual hospital trusts are publicly reported [21]. For the palliative treatment of lung cancer, seven out of 147 trusts had results above the 95\% upper control limit and two trusts even had results above the $99.8 \%$ control limit [8]. It is unknown whether public reporting will eventually lead to better results. Such an endeavour requires considerable data collection.

Our work has several limitations, which are mainly related to the limitations of the database. Information on performance score, comorbidity and other prognostic factors such as socioeconomic status and ethnicity was lacking, which limits the interpretability and generalisability of our data. Moreover, we only evaluated the start of treatment, while other studies assessed successive cycles in addition. Information on cause of death was not available, hampering distinction between death due to progressive disease or toxicity.

In conclusion, early mortality after the start of systemic treatment is a regular and inevitable incident for clinicians who treat patients with lung cancer. Prognostic factors have been identified, but these provide little support for decision making in individual cases. Additional data such as place and cause of death are needed to better appraise the value of the 30 -day mortality as a quality parameter.

Conflict of interest: J.A. Burgers reports having served on advisory boards for Boehringer Ingelheim, AstraZeneca and Roche outside the submitted work. R.A. Damhuis has nothing to disclose.

\section{References}

1 Spiro SG, Rudd RM, Souhami RL, et al. Chemotherapy versus supportive care in advanced non-small cell lung cancer: improved survival without detriment to quality of life. Thorax 2004; 59: 828-836.

2 Mok TS, Wu YL, Thongprasert S, et al. Gefitinib or carboplatin-paclitaxel in pulmonary adenocarcinoma. $N$ Engl J Med 2009; 361: 947-587.

3 Shepherd FA, Dancey J, Ramlau R, et al. Prospective randomized trial of docetaxel versus best supportive care in patients with non-small-cell lung cancer previously treated with platinum-based chemotherapy. J Clin Oncol 2000 18: 2095-2103.

4 Hellmann MD, Li BT, Chaft JE, et al. Chemotherapy remains an essential element of personalized care for persons with lung cancers. Ann Oncol 2016; 27: 1829-1835.

5 Masters GA, Temin S, Azzoli CG, et al. Systemic therapy for stage IV non-small-cell lung cancer: American Society of Clinical Oncology clinical practice guideline update. J Clin Oncol 2015; 33: 3488-3515.

6 Besse B, Adjei A, Baas P, et al. 2nd ESMO Consensus Conference on Lung Cancer: non-small-cell lung cancer first-line/second and further lines of treatment in advanced disease. Ann Oncol 2014; 25: 1475-1484.

7 Mak KS, van Bommel AC, Stowell C, et al. Defining a standard set of patient-centred outcomes for lung cancer. Eur Respir J 2016; 48: 852-860.

8 Wallington M, Saxon EB, Bomb M, et al. 30-day mortality after systemic anticancer treatment for breast and lung cancer in England: a population-based, observational study. Lancet Oncol 2016; 17: 1203-1216.

9 Bebb GCW, D'Silva A, Elegbede A, et al. 30-day mortality following systemic anti-cancer treatment for NSCLC at a single Canadian cancer centre. International Association for the Study of Lung Cancer World Conference on Lung Cancer. Yokohama, Japan, 2017.

10 Rochigneux P, Raoul JL, Beaussant Y, et al. Use of chemotherapy near the end of life: what factors matter? Ann Oncol 2017; 28: 809-817.

11 Stephens RJ, Girling DJ, Machin D. Treatment-related deaths in small cell lung cancer trials: can patients at risk be identified? Lung Cancer 1994; 11: 259-274.

12 Higginson IJ, Sen-Gupta GJ. Place of care in advanced cancer: a qualitative systematic literature review of patient preferences. J Palliat Med 2000; 3: 287-300.

13 Gomes B, Higginson IJ, Calanzani N, et al. Preferences for place of death if faced with advanced cancer: a population survey in England, Flanders, Germany, Italy, the Netherlands, Portugal and Spain. Ann Oncol 2012; 23 2006-2015.

14 Bekelman JE, Halpern SD, Blankart CR, et al. Comparison of site of death, health care utilization, and hospital expenditures for patients dying with cancer in 7 developed countries. JAMA 2016; 315: 272-283.

15 Le Caer H, Borget I, Corre R, et al. Prognostic role of a comprehensive geriatric assessment on the management of the elderly patients with advanced non-small cell lung cancer (NSCLC): a pooled analysis of two prospective phase II trials by the GFPC Group. J Thorac Dis 2017; 9: 3747-3754.

16 O'Brien ME, Borthwick A, Rigg A, et al. Mortality within 30 days of chemotherapy: a clinical governance benchmarking issue for oncology patients. Br J Cancer 2006; 95: 1632-1636.

17 Reck M, Rodríguez-Abreu D, Robinson AG, et al. Pembrolizumab versus chemotherapy for PD-L1-positive non-small-cell lung cancer. N Engl J Med 2016; 375: 1823-1833.

18 Hellmann MD, Ciuleanu TE, Pluzanski A, et al. Nivolumab plus ipilimumab in lung cancer with a high tumor mutational burden. N Engl J Med 2018; 378: 2093-2104.

19 Gandhi L, Rodríguez-Abreu D, Gadgeel S, et al. Pembrolizumab plus chemotherapy in metastatic non-small-cell lung cancer. N Engl J Med 2018; 378: 2078-2092.

20 Socinski MA, Jotte RM, Cappuzzo F, et al. Atezolizumab for first-line treatment of metastatic nonsquamous NSCLC. N Engl J Med 2018; 378: 2288-2301.

21 Wallington MS, Saxon EB, Bomb M, et al. Trust-level 30-day mortality after systemic anticancer treatment for breast and lung cancer in England. Companion report. London, Public Health England, 2016. 